\title{
Differentiating neural crest stem cells induce proliferation of cultured rodent islet beta cells
}

\author{
G. Grouwels • S. Vasylovska • J. Olerud • G. Leuckx • \\ A. Ngamjariyawat $\cdot$ Y. Yuchi $\cdot$ L. Jansson $\cdot$ \\ M. Van de Casteele $\cdot$ E. N. Kozlova $\cdot$ H. Heimberg
}

Received: 28 November 2011 / Accepted: 22 February 2012 / Published online: 23 May 2012

(C) Springer-Verlag 2012

\begin{abstract}
Aims/hypothesis Efficient stimulation of cycling activity in cultured beta cells would allow the design of new strategies for cell therapy in diabetes. Neural crest stem cells (NCSCs) play a role in beta cell development and maturation and increase the beta cell number in co-transplants. The mechanism behind NCSC-induced beta cell proliferation and the functional capacity of the new beta cells is not known. Methods We developed a new in vitro co-culture system that enables the dissection of the elements that control the cellular interactions that lead to NCSC-dependent increase in islet beta cells.
\end{abstract}

G. Grouwels and S. Vasylovska are joint first authors.

E.N. Kozlova and H. Heimberg are joint senior authors.

Electronic supplementary material The online version of this article (doi:10.1007/s00125-012-2542-0) contains peer-reviewed but unedited supplementary material, which is available to authorised users.

G. Grouwels · G. Leuckx • Y. Yuchi · M. Van de Casteele •

H. Heimberg $(\bowtie)$

Diabetes Research Center, Vrije Universiteit Brussel,

Laarbeeklaan 103,

B1090 Brussels, Belgium

e-mail: Harry.Heimberg@vub.ac.be

S. Vasylovska $\cdot$ J. Olerud $\cdot$ A. Ngamjariyawat • E. N. Kozlova $(\bowtie)$

Department of Neuroscience,

Uppsala University Biomedical Center,

Box 593, SE-751 24 Uppsala, Sweden

e-mail: Elena.Kozlova@neuro.uu.se

L. Jansson

Department of Medical Cell Biology,

Uppsala University Biomedical Center,

Uppsala, Sweden
Results Mouse NCSCs were cultured in vitro, first in medium that stimulated their proliferation, then under conditions that supported their differentiation. When mouse islet cells were cultured together with the NCSCs, more than $35 \%$ of the beta cells showed cycle activity. This labelling index is more than tenfold higher than control islets cultured without NCSCs. Beta cells that proliferated under these culture conditions were fully glucose responsive in terms of insulin secretion. NCSCs also induced beta cell proliferation in islets isolated from 1 -year-old mice, but not in dissociated islet cells isolated from human donor pancreas tissue. To stimulate beta cell proliferation, NCSCs need to be in intimate contact with the beta cells. Conclusions/interpretation Culture of islet cells in contact with NCSCs induces highly efficient beta cell proliferation. The reported culture system is an excellent platform for further dissection of the minimal set of factors needed to drive this process and explore its potential for translation to diabetes therapy.

Keywords Beta cell $\cdot$ Cell culture $\cdot$ Diabetes $\cdot$ Neural crest . Proliferation

$\begin{array}{ll}\text { Abbreviations } \\ \text { BB rat } & \text { BioBreeding rat } \\ \text { bFGF } & \text { Basic fibroblast growth factor } \\ \text { DIFF } & \text { Differentiation medium } \\ \text { DRG } & \text { Dorsal root ganglia } \\ \text { E } & \text { Embryonic day } \\ \text { EdU } & \text { 5-Ethynyl-2'-deoxyuridine } \\ \text { GFAP } & \text { Glial fibrillary acidic protein } \\ \text { GSIS } & \text { Glucose-stimulated insulin secretion } \\ \text { NCSC } & \text { Neural crest stem cell } \\ \text { PDX1 } & \text { Pancreatic and duodenal homeobox 1 } \\ \text { PROP } & \text { Propagation medium }\end{array}$




\section{Introduction}

The rich innervation of the endocrine pancreas contributes to the regulation of islet hormone secretion [1-3] and supports the islet cells with trophic signals [4]. In type 1 diabetes, islet innervation is affected by the autoimmune attack [5]. In BioBreeding (BB) rats, an experimental model for type 1 diabetes, these nerves disappear even before beta cells are lost [6]. Recent studies have demonstrated that neural crest stem cells (NCSCs), from which all neurons that innervate the islets are derived, play an important role in regulating beta cell mass during early development [7, 8] and during islet maturation [9]. We previously showed that mature sensory neurons influence insulin release by adult islets in vitro [10]. Furthermore, islets themselves induce migration and differentiation of NCSCs towards a neuronal phenotype both in vitro [10] and in vivo when the two cell types were transplanted under the kidney capsule [11]. Within a graft containing both NCSCs and islets, the beta cells display an increased proliferation [11]. In view of these findings, the current report describes the potential of NCSCs to induce beta cell proliferation in order to increase the glucose responsive beta cell mass. In the present study, we prepared NCSCs from the dorsal root ganglia (DRG) of embryonic (embryonic day [E] 11.5) mice. The DRG preparation included the boundary cap, a transient neural crestderived structure giving rise to the last wave of DRG neurons in development [12-14]. The NCSCs are multipotent stem cells that have been shown to generate sensory neurons in vitro $[15,16]$ and glial cells and neurons in vivo after transplantation $[17,18]$.

Here we show that NCSCs induce proliferation of beta cells from young adult and 1-year-old mice by direct contact, but fail do so in human beta cells. The proliferating beta cells retain their normal capacity to secrete insulin in response to increasing concentrations of glucose.

\section{Methods}

Mice, cell isolation and culture All animal experiments were performed according to the guidelines of the Vrije Universiteit Brussel Animal Studies Ethics Committee. Islets were isolated from 8 -week-old $\mathrm{Balb} / \mathrm{C}$ mice or from 8-week-old and 12-month-old C57 Black6 mice for aged islet experiments (Elevage Janvier, Le Genest Saint Isle, France). Pancreases were inflated with isolation medium (code BESP163Q; Lonza, Basel, Switzerland) containing $0.3 \mathrm{mg} / \mathrm{ml}$ collagenase (code C7657; Sigma-Aldrich, St Louis, MO, USA) by injection via the pancreatic duct. The pancreas was then dissected and further digested. Islets were hand-picked using glass Pasteur pipettes under the microscope and immediately placed in culture in RPMI 1640 (code 61870-010; Invitrogen, Carlsbad, CA, USA) with $11.11 \mathrm{mmol} / 1$ glucose. Human islets were harvested from three different donors: two 57-year-old women and one 27-year-old woman. Ethics approval to use endocrineenriched cells derived from donor organs was given by the Medical Ethics Committee of the University Hospital of the Vrije Universiteit Brussel (O.G. 016) to the Beta Cell Bank-University Hospital Brussels (permission 2010/193). Permission was also given to $\mathrm{H}$. Heimberg by the Ethics Committee to use these cells for in vitro studies aimed to develop improved transplantation conditions (2008/048).

NCSC cultures were prepared according to a previously published protocol [16]. The medium was changed every other day (50\% of the medium replaced with fresh medium) until neurospheres began to form. Neurospheres were then kept free-floating in propagation medium (PROP: DMEM/ F12 medium (code 31330-038; Invitrogen) supplemented with B27 (code 17504-044; Invitrogen), N2 (code 17502048; Invitrogen) and containing $20 \mathrm{ng} / \mathrm{ml}$ basic fibroblast growth factor (bFGF) (code 13256-029; Invitrogen) and $20 \mathrm{ng} / \mathrm{ml}$ epidermal growth factor (EGF) (code 236-EG; R\&D systems, Minneapolis, MN, USA). DIFF medium consisted of 50\% DMEM/F12 medium (code 31330-038; Invitrogen) and 50\% neurobasal (code 10888022; Invitrogen) supplemented with B27 and N2.

For co-culture of islets and neurospheres, 24-well plates were coated for $1 \mathrm{~h}$ at $37^{\circ} \mathrm{C}$ with $10 \mu \mathrm{g} / \mathrm{ml}$ poly-lysine (code P1399; Sigma-Aldrich) followed by washing with bidistilled water and subsequent coating with laminin (code L2020; Sigma-Aldrich) for at least $4 \mathrm{~h}$ at $37^{\circ} \mathrm{C}$ at a concentration of $10 \mu \mathrm{g} / \mathrm{ml}$. Neurospheres and pancreatic islets were seeded in the wells in drops of $15 \mu \mathrm{l}$ medium and left for $1 \mathrm{~h}$ at $37^{\circ} \mathrm{C}$ and $5 \% \mathrm{CO}_{2}$ to improve their attachment to the wells. The appropriate medium was then supplemented to the wells.

For transwell assays, cells were plated both on coverslips in the bottom of 24-well plates as well as in cell culture inserts (code 353095; BD Biosciences, Franklin Lakes, NJ, USA) with a $0.4 \mu \mathrm{m}$ pore size membrane separating the upper and lower compartments. Further culture conditions and manipulations are similar to what has been described above.

To measure the incorporation of the nucleoside analogue 5-ethynyl-2'-deoxyuridine (EdU) (code C10337; Invitrogen), EdU was added to the culture media at a final concentration of $10 \mu \mathrm{mol} / \mathrm{l}$.

Protein analysis Cells were fixed in 10\% buffered paraformaldehyde for 20 min then washed with PBS prior to permeabilisation for 15 min using $0.2 \%$ Triton X-100. After washing with PBS, cells were incubated for $30 \mathrm{~min}$ in normal donkey serum. Primary antibody was then applied overnight at $4{ }^{\circ} \mathrm{C}$. Sections were washed with PBS and incubated with a secondary antibody for $2 \mathrm{~h}$ at room 
temperature before washing again with PBS. For subsequent stainings, the protocol was repeated starting from the primary antibody step. DNA was labelled using Hoechst 33342 (Sigma-Aldrich) at a final concentration of $8 \mu \mathrm{g} / \mathrm{ml}$ for 20 min. Coverslips were then mounted on glass slides with vectashield fluorescent mounting medium (code h-1000; Vector laboratories, Burlingame, CA, USA).

Primary antibodies used: anti-insulin (guinea pig, gift from C. van Schravendijk, Diabetes Research Center, Vrije Universiteit Brussel), anti-glial fibrillary acidic protein (GFAP) (rabbit, code z0334; Dako, Glostrup, Denmark), anti- $\beta$-tubulin (mouse, code 32-2600; Invitrogen), antipancreatic duodenal homeobox 1 (PDX1) (goat, provided by the Beta Cell Biology Consortium) and anti-cleaved caspase 3 (Rabbit, code 9661 s; Cell Signaling, Beverly, MA, USA).

All secondary antibodies were purchased from the Jackson Immunoresearch Laboratories (West Grove, PA, USA) (Anti-guinea pig CY3, code 706-166-148; anti-rabbit CY2, code 711-486-152; anti-rabbit CY5, code 711-496-152; antimouse CY2, code 715-486-150; anti-mouse CY5, code 715495-150; and anti-goat CY3, code 705-165-003).

Staining for EdU was performed according to the manufacturer's recommendations. A $300 \mu \mathrm{l}$ aliquot of the staining solution was added to the wells and left in the dark for 30 min prior to washing with PBS. Neurite length was determined according to a previously published method [19].

Fluorescence microscopy was carried out on an Olympus BX61 microscope equipped with Olympus UPlan SApo lenses (Aartselaar, Belgium). Images were captured via an Orca $\mathrm{R}^{2} \mathrm{C} 10600$ digital camera (Hamatsu, Herrsching am Ammersee, Germany) and processed using Smartcapture software (Digital Scientific, Cambridge, UK). Light emission was using a Lumen 200 (Prior Scientific Instruments, Cambridge, UK) light emitter.

Confocal microscopy was conducted using a Zeiss LSM 710 NLO microscope (Carl Zeiss, Zaventem, Belgium) combined with a Mai-Tai deepsee multiphoton laser. Image analysis and manipulation was performed using Zen LE software (Carl Zeiss).

Insulin content and release were determined at the end of the culture period: following 3 days in PROP and 7 days in PROP or DIFF, the medium was replaced by RPMI 1640 and cells were left for an extra $24 \mathrm{~h}$ in culture. Glucose-stimulated insulin release assay and insulin content measurement were then performed as described previously [20].

Statistical analysis Data were expressed as means \pm SEM. Student's $t$ test or ANOVA with Bonferroni post hoc test were used for comparisons. Differences were considered statistically significant when $p<0.05$.

\section{Results}

Mouse pancreatic islets and NCSCs interact when cocultured Migration and differentiation of NCSCs are stimulated by co-culture with pancreatic islets [10]. Inspired by this finding, we examined the effect of NCSCs on islet cells when plated at a $1 / 1$ ratio at the start of co-culture. The composition of the individual components of the in vitro model was determined before co-culture: islets isolated from adult mouse pancreases and cultured for 3 days were characterised by the abundant presence of insulin ${ }^{+}$cells and contained no $\mathrm{GFAP}^{+}$and no $\beta$-tubulin class $\mathrm{III}^{+}$insulin mature neuronal cells (Fig. 1a). The neurospheres formed by NCSCs isolated from the DRG of E11.5 mice [16] were insulin $^{-}$(data not shown), proliferated and remained undifferentiated when cultured in PROP [10]. When cultured in DIFF, which differs from PROP by the absence of bFGF and EGF, or when subjected for 2 days to an in vitro differentiation assay [17], the cells differentiated into either $\mathrm{GFAP}^{+}$or $\beta$-tubulin III $^{+}$cells [10]. When islets and neurospheres were co-cultured in PROP for 1 week on glass slides coated with poly-lysine and laminin, axonal length was short (Fig. 1c). However, when NCSCs were co-cultured with islets in DIFF, $\beta$-tubulin $\mathrm{III}^{+}$neurites grew, on average, three times longer than in PROP (Fig. 1d,e). When islets were cocultured with NCSCs in PROP, few $\mathrm{GFAP}^{+}$or $\beta$-tubulin ${ }^{+}$ fibres contacted the beta cells in the core of the islets (Fig. 1f). However, when cultured for 3 days in PROP followed by 7 days in DIFF, a condition from now on referred to as PROP-DIFF, numerous $\beta$-tubulin $\mathrm{III}^{+}$neurites could be observed deep inside the islets where $\mathrm{GFAP}^{+}$fibres were rather rare (Fig. 1g). Z-stack images showed that these islets were surrounded by NCSCs and their derivatives under both conditions, PROP (electronic supplementary material [ESM] Fig. 1a) and PROP-DIFF (ESM Fig. 1b). NCSCs indeed have a strong capacity to migrate in vivo and in vitro $[8,10]$ and thus are able to migrate into the islets, making cell-cell contacts.

NCSCs induce proliferation of co-cultured primary islet beta cells NCSCs strongly increase islet cell proliferation when co-transplanted under the kidney capsule of mice [11]. These in vivo data did not distinguish between the direct effects of NCSCs on the islet cells and possible indirect effects through other, non-endocrine islet cell types, such as endothelial cells [21]. We therefore investigated the effect of NCSCs on islet beta cell proliferation in vitro. Beta cells were stained for insulin and the cells that were actively cycling were traced by immunostaining with the thymidine analogue EdU, which was incorporated in their DNA when added to the medium during the last 7 days of culture. Culture was in 24-well plates on cover slips coated with poly-lysine and laminin. The cells that were positive for 
a
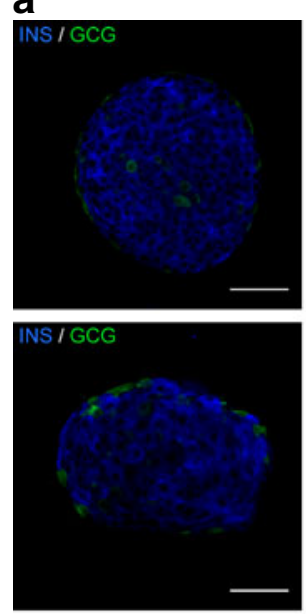

C

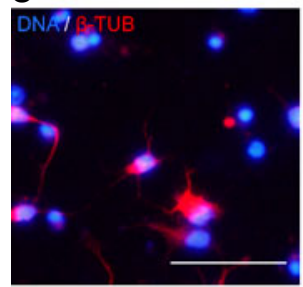

d
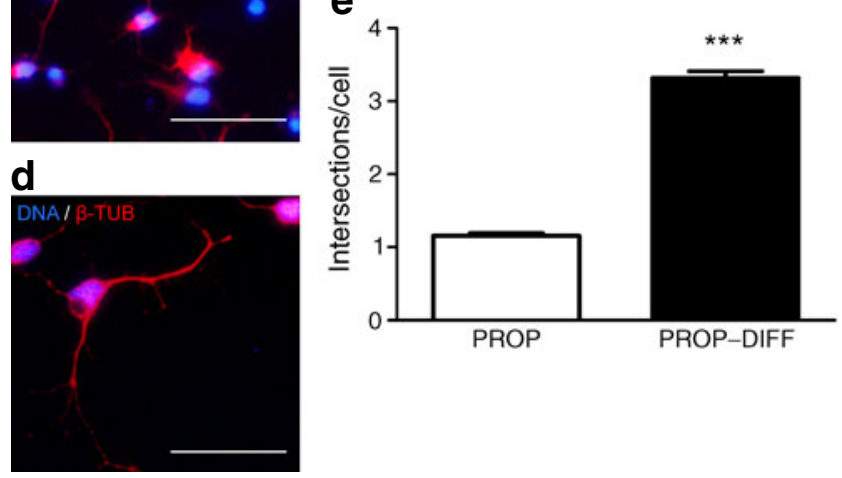

f
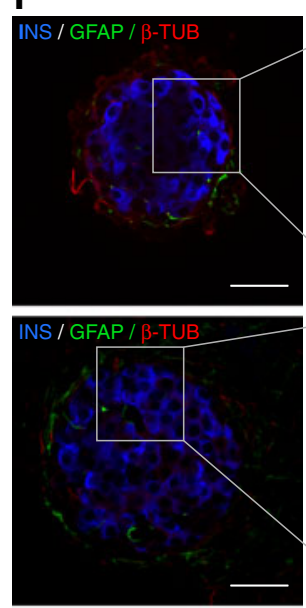

g
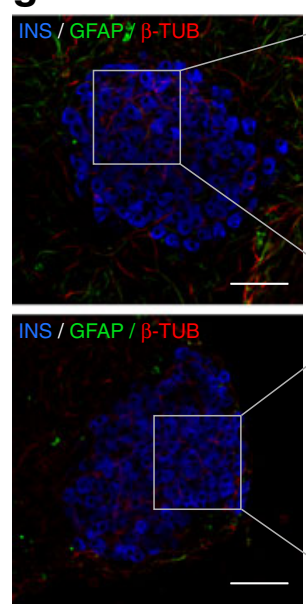
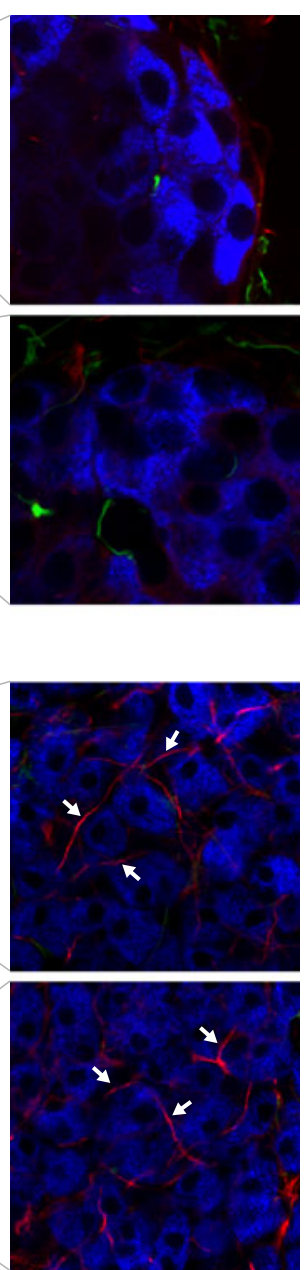

Fig. 1 Mouse pancreatic islets and NCSCs interact when co-cultured. (a) Isolated mouse pancreatic islets precultured for 3 days in RPMI medium were immunostained for insulin (INS, blue) and glucagon (GCG, green) in combination with either $\beta$-tubulin ( $\beta$-TUB) or GFAP (red). No GFAP ${ }^{+}$cells were observed in the islets and, although all beta cells expressed $\beta$-tubulin, no insulin ${ }^{-} \beta$-tubulin ${ }^{+}$cells were found. (b) Positive control for $\beta$-tubulin (blue) and GFAP (green) immunostaining was performed on a co-culture of NCSCs and islets in PROPDIFF. Neurite size from NCSCs increased when co-cultured with pancreatic islets in PROP-DIFF (c) compared with PROP (d)

both insulin and EdU were considered to be proliferating beta cells that had already divided or were still in the Sphase of the cell cycle. Islets or islet cells and NCSCs were cultured for 3 days in PROP to allow spreading and growth of NCSCs. This 3 day culture period was either continued in PROP or changed to DIFF (PROP-DIFF), for 7 days (Fig. 2a). When islets or islet cells were cultured without NCSCs in PROP or PROP-DIFF or when they were cocultured with NCSCs in either PROP or DIFF, little or no beta cell proliferation was observed. However, when cocultured with NCSCs in PROP-DIFF, the EdU-labelling index of islet beta cells dramatically increased more than tenfold (from $2.1 \pm 0.8 \%$ for islets only to $23.9 \pm 1.4 \%$ for
( $\beta$-tubulin: red; DNA: blue). (e) Quantification of the length of axons in PROP-DIFF vs PROP $(n=7 ; * * * p<0.001$, error bar is SEM). (f) After 1 week co-culture with NCSCs on poly-lysine- and laminincoated coverslips in PROP, GFAP ${ }^{+}$or $\beta$-tubulin ${ }^{+}$fibres surround the islets (see also ESM Fig. 1a,b) but only a few were inside the islets. In contrast, after 1 week co-culture in PROP-DIFF (g), many $\beta$-tubulin ${ }^{+}$ neurites were located deep inside the islets (arrows). GFAP ${ }^{+}$fibres mainly remained outside the islets. (f) and (g) are representative pictures. Scale bars represent $50 \mu \mathrm{m}$

islets in the presence of NCSCs (Fig. 2b,e). Among dissociated islet cells, up to $38.5 \pm 5.9 \%$ of the beta cells incorporated EdU in their nuclear DNA during co-culture with NCSCs in PROP-DIFF (Fig. 2c,g).

In order to investigate whether the increased beta cell proliferation also gave rise to an increase in the total beta cell mass, single dissociated islet cells were seeded with or without NCSCs on 96-well plates coated with poly-lysine and laminin and kept in culture as described above (Fig. 2a). At the end of culture, cells were fixed and stained for PDX1 in order to label all beta cell nuclei. Pictures of entire wells were taken and the total number of $\mathrm{PDX} 1^{+}$nuclei per well was manually counted. No statistically significant difference 


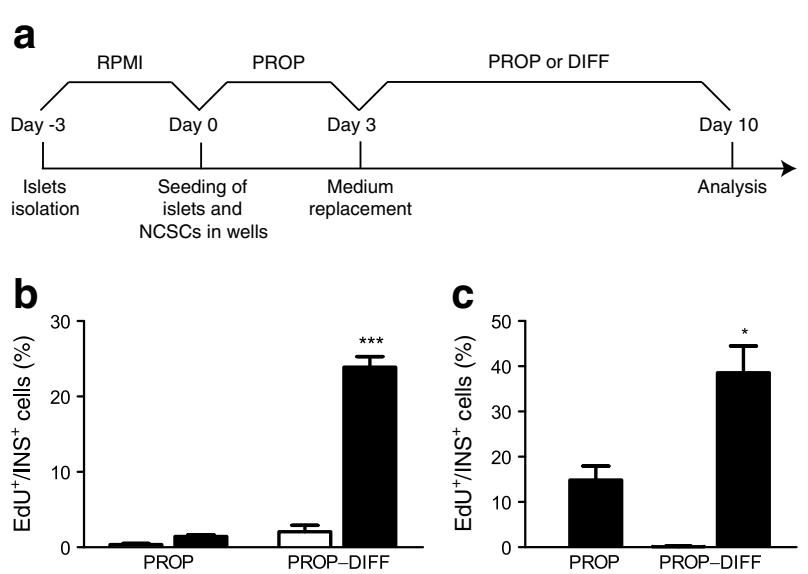

d
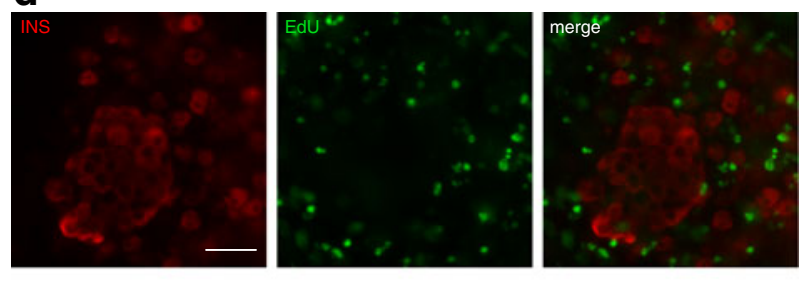

e
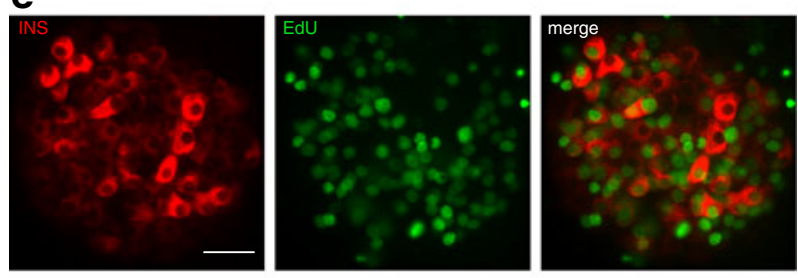

$\mathbf{f}$
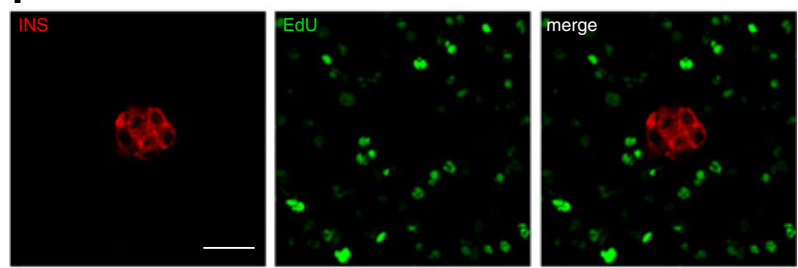

g
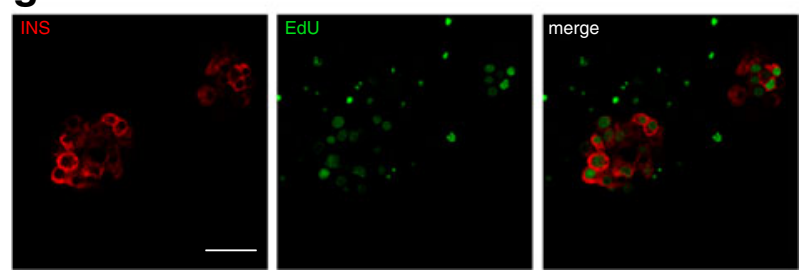

h

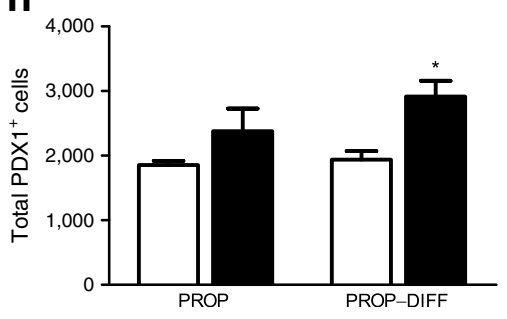

Fig. 2 NCSCs induce proliferation of co-cultured primary islet beta cells. (a) Schematic timeline of the different conditions analysed. (b,c) After culture either in PROP (10 days) or PROP-DIFF (3+7 days) with thymidine analogue (EdU) labelling for the last 7 days on polylysine- and laminin-coated coverslips, the level of proliferating beta cells is dramatically increased when co-cultured in PROP-DIFF compared with a culture without NCSCs or a co-culture in PROP. Proliferation of the beta cells in intact islets (b) and in dissociated islets (c) was significantly increased in PROP-DIFF co-culture $(n=3-9 ; * p<$ 0.05 and $* * * p<0.0001$ for PROP-DIFF vs PROP; white bars: intact or dissociated islets cultured without NCSCs; black bars: intact or dissociated islets cultured with NCSCs). Representative pictures of insulin (red) and EdU (green) detection by immunofluorescence in islets (d, e) and dissociated islet cells (f, g), co-cultured with NCSCs in PROP (d and f) or PROP-DIFF (e and $\mathbf{g}$ ). Scale bars represent $50 \mu \mathrm{m}$. (h) The number of beta cells under different experimental conditions was assayed by seeding single dissociated islet cells in 96-well plates coated with poly-lysine and laminin (white bars: single cells cultured without NCSCs; black bars: single cells cultured with NCSCs). Staining for nuclear PDX1 was performed before picturing the entire wells. Manual counting of all PDX1 $1^{+}$nuclei provided an objective assessment of the difference in total beta cell number $\left(n=3-5 ;{ }^{*} p<0.05\right.$ for PROP-DIFF without vs with NCSCs)

could be observed between single cells cultured in PROP with or without NCSCs $(1856 \pm 61$ cells without NCSCs vs $2377 \pm 351$ cells with NCSCs). When single cells were cocultured with NCSCs in PROP-DIFF, a significant increase in the total $\mathrm{PDX}^{+}$cells could be observed when compared with single cells cultured in PROP-DIFF without NCSCs $(1939 \pm 133$ cells in PROP-DIFF alone vs $2916 \pm 241$ cells in PROP-DIFF with NCSCs) (Fig. 2h).

Beta cells from aged mice, but not human beta cells, have the capacity to proliferate We next investigated whether the co-culture of islets and NCSCs could induce proliferation in beta cells isolated from either aged mice or human donor pancreases, two models known for poor, if any, cycling activity. Islet beta cells isolated from aged mice (12 months old) are still able to enter the cell cycle when co-cultured under the conditions depicted above. Islets from aged and young ( 8 weeks old) mice were co-cultured with NCSCs in PROP and PROP-DIFF. EdU was provided to the cells for the last 7 days of culture and EdU incorporation by beta cells was assayed by immunocytochemistry. No proliferation was observed in beta cells of young or old islets cocultured with NCSCs in PROP. However, both young and old beta cells similarly incorporated EdU when co-cultured with NCSCs in PROP-DIFF ( $23 \pm 2 \%$ for young islets and $20 \pm 2 \%$ for old islets) (Fig. 3a). Islets from three human donor pancreases were isolated and cultured in PROP or PROP-DIFF both in the presence or absence of NCSCs as described above. EdU incorporation by beta cells during the last 7 days of co-culture remained below $0.3 \%$ under all conditions (Fig. 3b).

Beta cell proliferation decreased while death increased during co-culture To investigate the kinetics of beta cell 
a

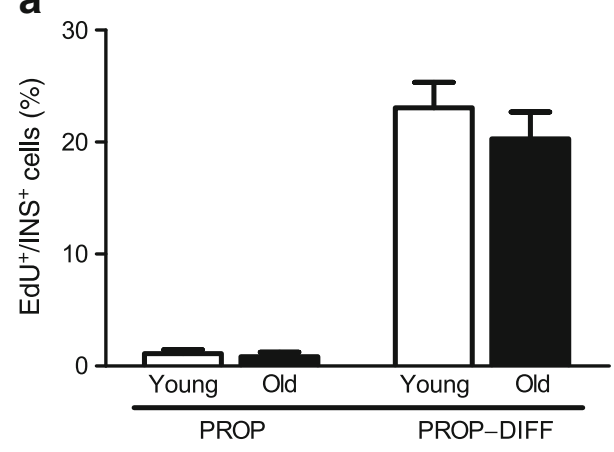

b

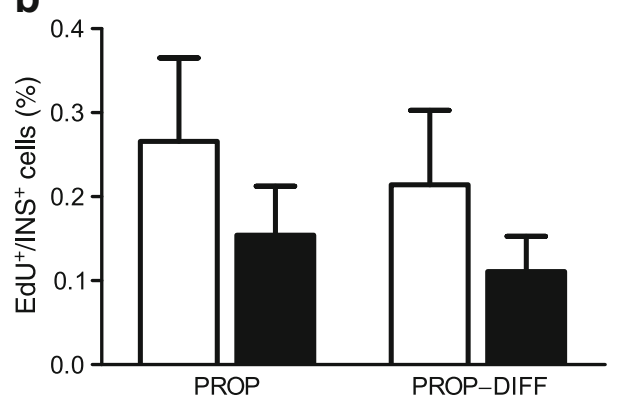

Fig. 3 Beta cells from aged mice, but not human beta cells have the capacity to proliferate. (a) Islets isolated from 8-week-old mice and from 12-month-old mice were co-cultured with NCSCs in PROP or PROP-DIFF as described above (see Fig. 2a). While no, or very few, proliferating beta cells could be observed in either young and old islets when co-cultured with NCSCs in PROP $(1.1 \pm 0.4 \%$ and $0.9 \pm 0.4 \%$, respectively), the proportion of proliferating beta cells in PROP-DIFF increased similarly to what has been seen in our earlier experiments (see Fig. 2b,c) in both young and old islets $(23 \pm 2.3 \%$ and $20.3 \pm 2.4 \%$, respectively) ( $n=3)$. (b) Dissociated islets obtained from three different donors were co-cultured with NCSCs as described above. Beta cell proliferation assayed by EdU incorporation during the last 7 days of culture remained hardly detectable in all conditions $(0.3 \pm 0.1 \%$ and $0.1 \pm$ $0.06 \%$ for human islets cells in PROP with and without NCSCs, respectively; $0.2 \pm 0.09 \%$ and $0.1 \pm 0.04 \%$ for human islet cells in PROP-DIFF with and without NCSCs, respectively). White bars represent a culture without NCSCs, and black bars a culture with NCSCs

proliferation and apoptosis during co-culture with NCSCs, islets were precultured for 3 days in RPMI prior to seeding in 24 well plates with NCSCs and kept in PROP medium for 3 days. Medium was then replaced by either PROP or DIFF and cells were kept in culture for 7, 11, 15 or 18 days in total. EdU was added to the culture for the last 3 days and immune-detected, together with insulin and activated caspase 3 , at fixation to find out when beta cell proliferation and apoptosis occur after induction of NCSC differentiation (Fig. 4a). Beta cell proliferation decreased between day 15 and day 18 of culture (Fig. 4b) while the amount of cleaved caspase $3^{+}$beta cells remained low both in PROP and PROP-DIFF until day 11 , then increased at day 15 in PROP-DIFF and finally increased further both in PROP a
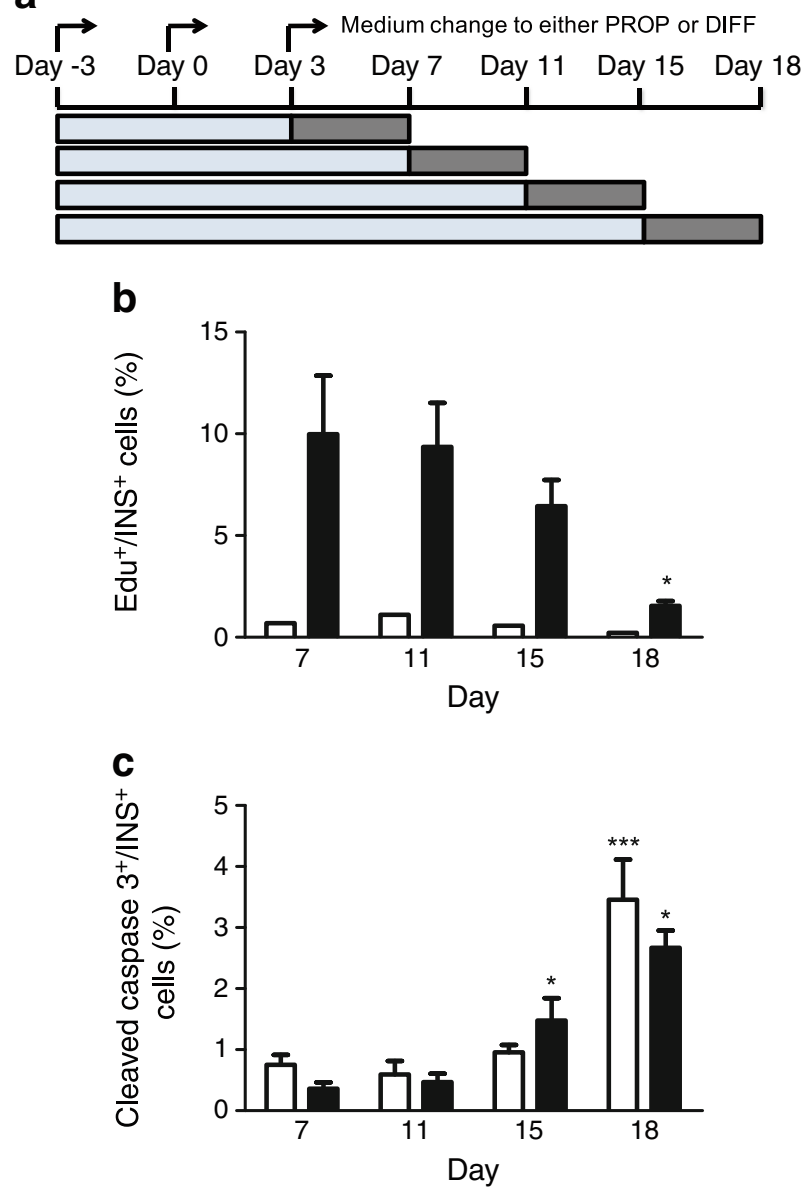

Fig. 4 Beta cell proliferation and cell death follow opposite trends. (a) Schematic diagram of the protocol used to study the kinetics of beta cell proliferation as well as beta cell apoptosis during co-culture with NCSCs. Cells were cultured as previously described until day 3. Dark grey represents the inclusion of EdU in the culture medium. Cells were fixed for analysis of proliferation (b) and apoptosis (c) at day 7, day 11, day 15 and day 18. For each time point, EdU was provided for a period of 3 days prior to fixation. Cleaved caspase 3 and insulin immunostaining assessed beta cell apoptosis at the different time points $(n=4$; ${ }^{*} p<0.05 * * * p<0.001$ vs corresponding condition at the previous time point). For both graphs, white bars represent a co-culture of islets and NCSCs in PROP and black bars a co-culture in PROP-DIFF

and PROP-DIFF at day 18 (Fig. 4c). Beta cell proliferation and death thus follow opposite trends during co-culture.

Proliferating beta cells remain glucose responsive The total insulin content of the islets alone or with NCSCs in PROP or PROP-DIFF was compared with islets cultured for 10 days in RPMI 1640, as a control condition [22]. Compared with islets in RPMI 1640, islets alone or in the presence of NCSCs cultured in PROP did not differ in total insulin content, while when cultured in PROP-DIFF they contained more insulin at the end of the experiment. These differences were, however, not statistically significant. Despite their increased beta cell proliferation, islets cultured 
with NCSCs in PROP-DIFF did not display a statistically significant increase in insulin content compared with the other conditions under study (Fig. 5a). As a control for a potential contribution of the culture medium to the presence of insulin in NCSCs, these cells were cultured in PROP or PROP-DIFF and found to be insulin-free (data not shown).

To assay whether islets are still functional after co-culture with NCSCs, glucose-stimulated insulin secretion (GSIS)
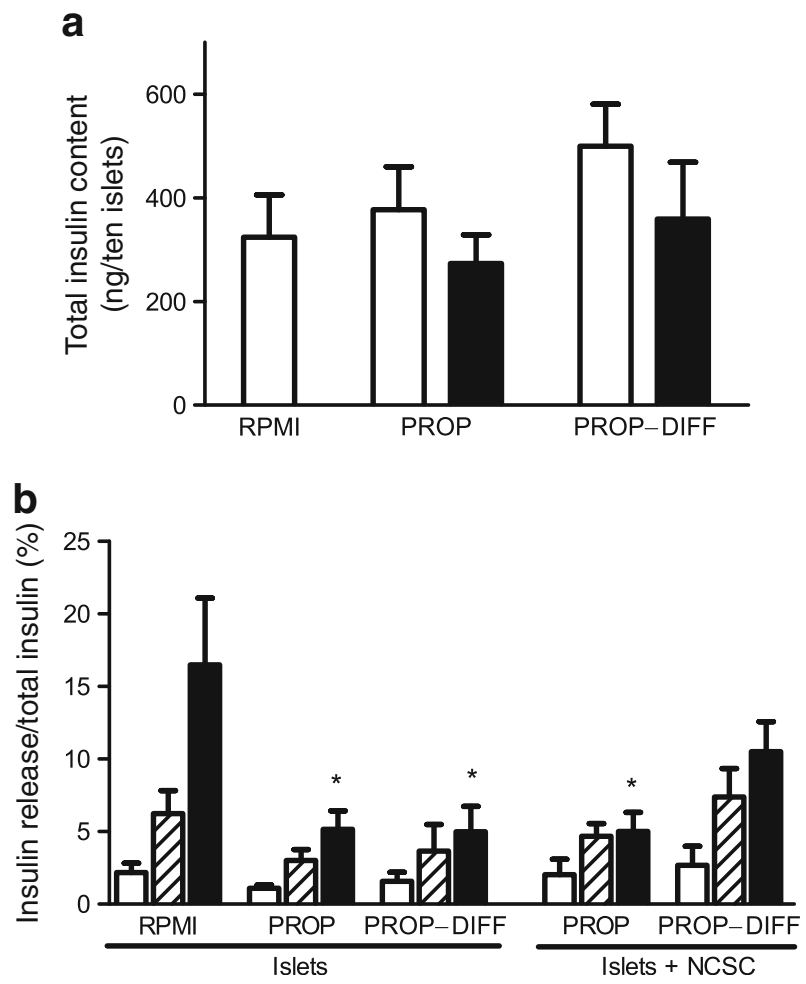

Fig. 5 Proliferating beta cells remain glucose responsive. (a) Islets were cultured in the usual RPMI 1640 medium or in PROP or PROPDIFF with and without NCSCs. After culture, the total insulin content or GSIS was measured. For both assays, a $24 \mathrm{~h}$ incubation period in RPMI 1640 was introduced in order to stabilise insulin production and secretion. For total insulin content, cells were washed with dissociation medium prior to lysis of the cells and measurement of insulin. No significant differences in total insulin content could be observed under any of the conditions studied, not even under the condition that induced high beta cell proliferation $(n=4, p>0.3)$. White bars represent a culture without NCSCs and black bars a culture with NCSCs. (b) For insulin secretion, cells were incubated in a medium containing no glucose for $30 \mathrm{~min}$ to set the basal insulin release and then medium was changed to 2 (white bars), 10 (hatched bars) or 20 (black bars) $\mathrm{mmol} / \mathrm{l}$ glucose medium and cells were left in culture for a further $2 \mathrm{~h}$. At the end of this period, cells were washed and insulin content was determined, and the $2 \mathrm{~h}$ insulin release was expressed as a percentage of the insulin content. No statistical differences in GSIS were observed between any of the conditions tested, either at 2 or at $10 \mathrm{mmol} / \mathrm{lglu}-$ cose. At $20 \mathrm{mmol} / \mathrm{lglucose}$, islets in PROP or PROP-DIFF, as well as islets co-cultured with NCSCs in PROP, showed less glucose responsiveness in insulin release compared with islets co-cultured with NCSCs in PROP-DIFF $(n=4-6, * p<0.05)$, while there was no significant difference between islets co-cultured with NCSCs in PROPDIFF and control islets cultured in RPMI 1640 was measured. Islets were kept in RPMI 1640 (11.11 mmol glucose/l), PROP or PROP-DIFF as well as co-cultured with NCSCs either in PROP or PROP-DIFF. Because of high glucose concentrations being present in PROP (17.51 mmol/1) and DIFF (21.25 mmol/1), after culture for 10 days the media were changed to RPMI 1640 and $24 \mathrm{~h}$ of culture in this medium allowed the beta cells to return to their functional baseline prior to assaying GSIS. Following $30 \mathrm{~min}$ in glucose-free medium, the cells were stimulated for $2 \mathrm{~h}$ by 2,10 or $20 \mathrm{mmol} / \mathrm{l}$ glucose. Finally, the insulin was measured in the medium as well as in the cells and released insulin was expressed as a percentage of total insulin. None of the culture conditions affected GSIS either at 2 or at $10 \mathrm{mmol} / \mathrm{l}$ glucose $(n=4-6, p>0.05$ when comparing islets without or with NCSCs in PROP or PROP-DIFF) while GSIS at $20 \mathrm{mmol} / 1$ glucose was higher under all conditions tested $(p<0.05)$ (Fig. 5b). At $20 \mathrm{mmol} / 1$ glucose, only the islets in the presence of NCSCs and cultured in PROP-DIFF showed normal glucose responsiveness in terms of insulin secretion ( $n=4-6, p>0.05$ vs control islets). Moreover, a statistically significant difference could be observed in islets cultured in PROP or PROP-DIFF as well as co-cultured in PROP, when compared with co-culture in PROP-DIFF $(n=4-6, p<0.05)$. Thus, under conditions that stimulated beta cell proliferation, that is, in co-culture with NCSCs and in PROP-DIFF, GSIS was similar to islets cultured in normal control RPMI 1640 medium and higher than in all other conditions. These data show that the full capacity of insulin storage and GSIS was retained in proliferating beta cells.

Direct cell-cell interaction with NCSCs is needed to induce beta cell proliferation To direct future research for factors driving beta cell proliferation stimulated by NCSCs we investigated the requirements for cellular contact with NCSCs to activate the beta cell cycle. Islets were plated in the bottom compartment of a transwell assay and NCSCs in the upper compartment or the insert. The membrane separating the two compartments has a pore size of $4 \mu \mathrm{m}$, which allows molecules to migrate easily between compartments. Co-culture of islets and NCSCs separated from each other in PROP or in PROP-DIFF with EdU labelling during the last 7 days did not induce increased beta cell proliferation (Fig. 6). When islets were co-cultured with NCSCs in PROP-DIFF in the lower compartment (positive control), beta cell proliferation was stimulated similarly to that in Fig. 2b (data not shown). Co-culture of islets and NCSCs in PROP (negative control) did not stimulate beta cell proliferation (data not shown).

In order to find out whether beta cell proliferation was induced by soluble factors produced by beta cells when in contact with NCSCs in PROP-DIFF, the two cell types were cultured together in the upper compartment of a transwell plate, while islets only were plated in the bottom 


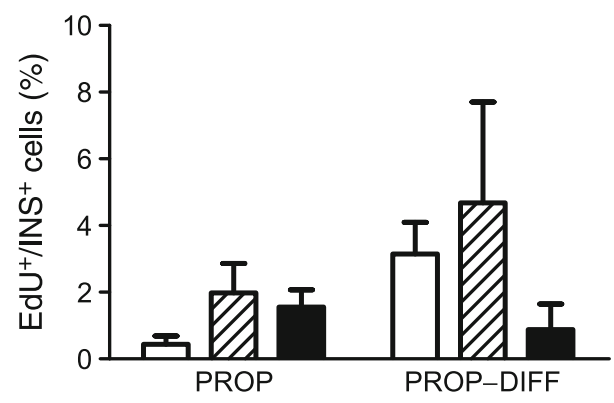

Fig. 6 Direct cell-cell interaction with NCSCs is needed to induce beta cell proliferation. Islets and NCSCs were cultured separated by a permeable membrane. Islets were confined to the lower compartment and NCSCs to the upper compartment of the transwell. The $4 \mu \mathrm{m}$ membrane pores allowed secreted molecules to diffuse between the two compartments. After culture either in PROP or PROP-DIFF with the thymidine analogue EdU present for the last 7 days, none of the experimental conditions induced beta cell proliferation. When islets and NCSCs were co-cultured in the upper compartment, no beta cells proliferated in the lower compartment that contained islets only ( $n=3-4$, $p>0.1$ for all comparisons). White bars indicate an empty insert, hatched bars an insert containing NCSCs and black bars, an insert containing a coculture of islets and NCSCs

compartment. Under these conditions, no beta cell proliferation was observed in the bottom of the transwell (Fig. 6). We thus concluded that NCSCs and islets need to be in direct contact in order to stimulate proliferation in beta cells, and that secreted factors present in the culture medium of NCSCs either alone or together with islets do not induce the beta cell cycle.

\section{Discussion}

The current report shows that early differentiating NCSCderived neural cells stimulate proliferation of islet beta cells isolated from old or young mice in co-culture, through direct cell-cell contact, and induce an increase in beta cell number. The resulting beta cells show normal glucose-stimulated insulin release. These findings extend our previous in vivo observations of beta cell proliferation in mouse islets cotransplanted with NCSCs [11]. However, the cellular interactions underlying this proliferative stimulus were unclear in the complex in vivo environment. We therefore developed an in vitro model for co-culture of NCSCs and islet cells. Within 10 days of co-culture, between $20 \%$ and $40 \%$ of islet beta cells had incorporated nucleoside analogues and thus were considered to be in the active phase of the cell cycle. This labelling index is much higher than any previously reported one [23], indicating that NCSCs are endowed with a so far unique ability to promote the beta cell cycle.

In previous reports, induction of mouse beta cell proliferation in vitro $[20,24,25]$ was by genetic manipulation using recombinant viruses to ectopically express, overexpress or silence target genes. The present model differs by its simplicity since it does not alter the genome of the beta cells in order to make them divide, but only needs specific stimuli from NCSCs. Imaging of NCSCs and islets cocultured in PROP revealed contacts between the two cell types within hours, but no beta cell proliferation was observed under these conditions. Cell-cell contact with NCSCs is thus, however, not sufficient to drive beta cell proliferation. Pronounced proliferation of beta cells was only seen following the withdrawal of mitogens from the medium, which causes the NCSCs to terminate proliferation and differentiate towards neuronal and glial cells (PROPDIFF conditions). This feature appears specific for early post-mitotic NCSCs, since sensory neurons co-cultured with islets failed to induce islet cell proliferation [26]. The in vitro interactions between NCSCs and islets resemble our previous observations of NCSC differentiation towards neuronal cells in the islet microenvironment [10], their migration in vivo and the formation of cell-cell interactions with islet cells after transplantation [11].

Evidence for age-related decrease in beta cell proliferation has accumulated both in mice [27-30] and humans [31]. More knowledge about the mechanism underlying this phenomenon might provide perspectives for the treatment of diabetes in aged patients [32]. We show that mouse beta cells isolated from 1-year-old mice and co-cultured with NCSCs demonstrate the same proliferative capacity as beta cells isolated from 8-week-old mice, demonstrating that exogenous factors are able to overcome the endogenous quiescence. Our co-culture protocol thus offers a platform to study beta cell proliferation under conditions of ageing.

The mechanism(s) underlying the stimulating effect on beta cell proliferation in NCSC-islet co-cultures are still obscure. Neurotrophic factors, including brain-derived neurotrophic factor [33], nerve growth factor [34], ciliary neurotrophic factor [35] and glial cell line derived neurotrophic factor [36] have been shown to promote survival and proliferation of beta cells. Our findings do not exclude a contribution of such secreted factor(s) to the observed beta cell proliferation but the result of the transwell assay demonstrates that they are not sufficient to drive beta cell cycling. Nonetheless, neurotrophic and other growth factors produced by NCSC-derived neurons and/or glia may play a role in the long-term functional maintenance of newly formed beta cells. The endocrine pancreas is normally richly innervated with sympathetic, parasympathetic, sensory, peptidergic and nitric oxide synthase containing neurons that help to modulate hormone secretion [1-3]. In rodent models, signals from the neural crest are important in regulating beta cell mass during development $[7,8]$ and neural signals are involved in the adaptive increase of beta cell mass in obesity [37]. There are several studies suggesting that neurotrophins, such as nerve growth factor and brain-derived neurotrophic factor, may affect the growth of both nerves and beta cells in 
mice $[4,38]$. Interestingly, not only beta cells but also nerve structures seem to be affected by the autoimmune assault seen during type 1 diabetes, and nerves disappear before any loss of beta cells occurs in type 1 diabetic BB rats [5, 6], which may hamper beta cell regenerative capacity. Thus, NCSCcontrolled expansion of a fully functional beta cell mass in vitro may at least in part recapitulate the role of the innervated microenvironment in the pancreas, needed for embryonic development and mature function of beta cells.

We were able to scale up the system to a co-culture of 300 islets with 300 neurospheres, followed by the isolation of the proliferated beta cells by fluorescence-activated cell sorting based on their endogenous characteristics, such as auto-fluorescence and granulation (ESM Fig. 2). This feature will allow us to test the potential of beta cells, purified following NCSC-induced expansion in vitro, to normalise glycaemia in diabetic mice following transplantation. The essentials of this model are easy to obtain. Indeed, NCSCs are not only present during development, they also persist in adulthood within the stem cell niche of hair follicles [39, 40] from which they could be harvested using a minimally invasive procedure and potentially serving autologous cell therapy. Alternatively, human embryonic stem cells could be differentiated into NCSCs [41]. In spite of the incapacity of mouse NCSCs to induce human beta cell proliferation, the current model that characterises conditions that expand functional beta cells could be a major step towards the identification of human-specific factors that might allow up-scaling of cellbased therapy for treatment of diabetic patients. Furthermore, the use of human-derived NCSCs might induce human beta cell proliferation when co-cultured in vitro.

Acknowledgements Special thanks to J. Coppens, A. Demarré, V. Laurysens, K. Sterck, G. Stangé, S. van Langenhoven and E. Quartier from the Diabetes Research Center of the Vrije Universiteit Brussel for technical assistance.

Funding Financial support was from the VUB Research Council $(\mathrm{HH})$, the Institute for the Promotion of Innovation by Science and Technology in Flanders (IWT) (GG and HH), the Swedish Research Council (project 20716), Stiftelsen Olle Engkvist Byggare, Signhild Engkvist Stiftelse, the Swedish Institute's Visby programme Dnr 00613/2011 (EK) and the Uppsala University Medical Faculty Fellowship Program (JO).

Duality of interest The authors declare that there is no duality of interest associated with this manuscript

\footnotetext{
Author contributions GG and SV acquired and analysed data and wrote the manuscript; JO, GL, YY and AN acquired and analysed data and revised the manuscript: LJ provided critical reading of the manuscript and designed experiments; MVDC designed experiments, acquired data and provided critical reading of the manuscript; ENK and $\mathrm{HH}$ designed experiments and wrote the manuscript. All authors approved the final version of the manuscript.
}

\section{References}

1. Brunicardi FC, Shavelle DM, Andersen DK (1995) Neural regulation of the endocrine pancreas. Int J Pancreatol 18:177-195

2. Ahrén B (2004) Sensory nerves contribute to insulin secretion by glucagon-like peptide-1 in mice. Am J Physiol Regul Integr Comp Physiol 286:R269-R272

3. Ahrén B (2000) Autonomic regulation of islet hormone secretionimplications for health and disease. Diabetologia 43:393-410

4. Teitelman G, Guz Y, Ivkovic S, Ehrlich M (1998) Islet injury induces neurotrophin expression in pancreatic cells and reactive gliosis of peri-islet Schwann cells. J Neurobiol 34:304-318

5. Winer S, Tsui H, Lau A et al (2003) Autoimmune islet destruction in spontaneous type 1 diabetes is not beta-cell exclusive. Nat Med 9:198-205

6. Mei Q, Mundinger TO, Lernmark A, Taborsky GJ Jr (2002) Early, selective, and marked loss of sympathetic nerves from the islets of BioBreeder diabetic rats. Diabetes 51:2997-3002

7. Plank JL, Mundell NA, Frist AY et al (2011) Influence and timing of arrival of murine neural crest on pancreatic beta cell development and maturation. Dev Biol 349:321-330

8. Nekrep N, Wang J, Miyatsuka T, German MS (2008) Signals from the neural crest regulate beta-cell mass in the pancreas. Development 135:2151-2160

9. Burris RE, Hebrok M (2007) Pancreatic innervation in mouse development and beta-cell regeneration. Neuroscience 150:592602

10. Kozlova EN, Jansson L (2009) Differentiation and migration of neural crest stem cells are stimulated by pancreatic islets. Neuroreport 20:833-888

11. Olerud J, Kanaykina N, Vasylovska S et al (2009) Neural crest stem cells increase beta cell proliferation and improve islet function in co-transplanted murine pancreatic islets. Diabetologia 52:2594-2601

12. Marmigere F, Ernfors P (2007) Specification and connectivity of neuronal subtypes in the sensory lineage. Nat Rev Neurosci 8:114 127

13. Maro GS, Vermeren M, Voiculescu O et al (2004) Neural crest boundary cap cells constitute a source of neuronal and glial cells of the PNS. Nat Neurosci 7:930-938

14. Ma Q, Fode C, Guillemot F, Anderson DJ (1999) Neurogenin1 and neurogenin2 control two distinct waves of neurogenesis in developing dorsal root ganglia. Genes Dev 13:1717-1728

15. Andang M, Hjerling-Leffler J, Moliner A et al (2008) Histone $\mathrm{H} 2 \mathrm{AX}$-dependent GABA(A) receptor regulation of stem cell proliferation. Nature 451:460-464

16. Hjerling-Leffler J, Marmigere F, Heglind M et al (2005) The boundary cap: a source of neural crest stem cells that generate multiple sensory neuron subtypes. Development 132:2623-2632

17. Aldskogius H, Berens C, Kanaykina N et al (2009) Regulation of boundary cap neural crest stem cell differentiation after transplantation. Stem Cells 27:1592-1603

18. Aquino JB, Hjerling-Leffler J, Koltzenburg M, Edlund T, Villar MJ, Ernfors P (2006) In vitro and in vivo differentiation of boundary cap neural crest stem cells into mature Schwann cells. Exp Neurol 198:438-449

19. Ronn LC, Ralets I, Hartz BP et al (2000) A simple procedure for quantification of neurite outgrowth based on stereological principles. J Neurosci Methods 100:25-32

20. Grouwels G, Cai Y, Hoebeke I et al (2010) Ectopic expression of E2F1 stimulates beta-cell proliferation and function. Diabetes 59:1435-1444

21. Olsson R, Carlsson PO (2006) The pancreatic islet endothelial cell: emerging roles in islet function and disease. Int J Biochem Cell Biol 38:492-497 
22. Andersson A (1978) Isolated mouse pancreatic islets in culture: effects of serum and different culture media on the insulin production of the islets. Diabetologia 14:397-404

23. Andersson A, Carlsson PO, Carlsson C et al (2004) Promoting islet cell function after transplantation. Cell Biochem Biophys 40:5564

24. Schisler JC, Fueger PT, Babu DA et al (2008) Stimulation of human and rat islet beta-cell proliferation with retention of function by the homeodomain transcription factor Nkx6.1. Mol Cell Biol 28:3465-3476

25. Fiaschi-Taesch NM, Salim F, Kleinberger J et al (2010) Induction of human beta-cell proliferation and engraftment using a single G1/S regulatory molecule, cdk6. Diabetes 59:1926-1936

26. Kozlova EN, Jansson L (2005) In vitro interactions between insulin-producing beta cells and embryonic dorsal root ganglia. Pancreas 31:380-384

27. Teta M, Long SY, Wartschow LM, Rankin MM, Kushner JA (2005) Very slow turnover of beta-cells in aged adult mice. Diabetes 54:2557-2567

28. Rankin MM, Kushner JA (2009) Adaptive beta-cell proliferation is severely restricted with advanced age. Diabetes 58:1365-1372

29. Rankin MM, Kushner JA (2010) Aging induces a distinct gene expression program in mouse islets. Islets 2:345-352

30. Tschen SI, Dhawan S, Gurlo T, Bhushan A (2009) Age-dependent decline in beta-cell proliferation restricts the capacity of beta-cell regeneration in mice. Diabetes 58:1312-1320

31. Perl S, Kushner JA, Buchholz BA et al (2010) Significant human beta-cell turnover is limited to the first three decades of life as determined by in vivo thymidine analog incorporation and radiocarbon dating. J Clin Endocrinol Metab 95:E234-E239
32. Chen H, Gu X, Liu Y et al (2011) PDGF signalling controls agedependent proliferation in pancreatic beta-cells. Nature 478:349-355

33. Yamanaka M, Itakura Y, Inoue T et al (2006) Protective effect of brain-derived neurotrophic factor on pancreatic islets in obese diabetic mice. Metabolism 55:1286-1292

34. Miao G, Mace J, Kirby M et al (2006) In vitro and in vivo improvement of islet survival following treatment with nerve growth factor. Transplantation 81:519-524

35. Rezende LF, Stoppiglia LF, Souza KL, Negro A, Langone F, Boschero AC (2007) Ciliary neurotrophic factor promotes survival of neonatal rat islets via the BCL-2 anti-apoptotic pathway. J Endocrinol 195:157-165

36. Mwangi S, Anitha M, Mallikarjun C et al (2008) Glial cell linederived neurotrophic factor increases beta-cell mass and improves glucose tolerance. Gastroenterology 134:727-737

37. Imai J, Katagiri H, Yamada T et al (2008) Regulation of pancreatic beta cell mass by neuronal signals from the liver. Science 322:1250-1254

38. Reimer MK, Mokshagundam SP, Wyler K, Sundler F, Ahren B, Stagner JI (2003) Local growth factors are beneficial for the autonomic reinnervation of transplanted islets in rats. Pancreas 26:392-397

39. Sieber-Blum M, Grim M, Hu YF, Szeder V (2004) Pluripotent neural crest stem cells in the adult hair follicle. Dev Dyn 231:258-269

40. Krejci E, Grim M (2010) Isolation and characterization of neural crest stem cells from adult human hair follicles. Folia Biol (Praha) $56: 149-157$

41. Chimge NO, Bayarsaihan D (2010) Generation of neural crest progenitors from human embryonic stem cells. J Exp Zool B Mol Dev Evol 314:95-103 